\title{
Antimicrobial Treatment of Orthopedic Implant-related Infections with Rifampin Combinations
}

\author{
Andreas F. Widmer, ${ }^{*}$ Andre Gaechter, Peter E. Ochsner, \\ and Werner Zimmerli
}

From the Divisions of Infectious Diseases and Orthopedic Surgery, University Hospital, Basel; and the Clinic of Orthopedic Surgery, Kantonsspital, Liestal, Switzerland

\begin{abstract}
The purpose of this prospective clinical study is to evaluate the role of combination chemotherapy with rifampin in the treatment of orthopedic device-related infections in which the implant could not be removed. Eleven patients with orthopedic implant-related infections due to staphylococci or streptococci were treated with the implant in situ. Each antimicrobial regimen included rifampin in combination with a $\beta$-lactam antibiotic or ciprofloxacin. The median duration of treatment with rifampin was 86 days (range, 15-336 days) with a median follow-up of $>24$ months after cessation of therapy. Treatment was successful for $82 \%$ of patients. Failures were associated with documented inappropriate treatment. These preliminary clinical data are supported by data from in vitro studies and animal experiments. Combination therapy with rifampin, in particular rifampin and a quinolone, should be considered for patients with orthopedic implant-related infections if the implant cannot be removed.
\end{abstract}

Long-term intravenous therapy combined with a one- or two-stage replacement is frequently necessary for controlling orthopedic device-related infection (ODRI) [1]. New antimicrobial regimens may cure infections that were previously considered as necessitating removal of the device [2]. We previously observed in an animal model that even short-term treatment with rifampin can sterilize implant-related infections due to Staphylococcus epidermidis and Staphylococcus aureus [3-5]. The present trial was designed as a pilot study for evaluating whether a rifampin-containing regimen could cure ODRIs rather than only suppress the clinical symptoms. We applied such a regimen to 11 patients who had ODRIs and from whom the device could not be removed.

We prospectively observed 11 patients (five women and six men) with ODRIs due to gram-positive cocci who were consulted by physicians from the Division of Infectious Diseases (A.F.W. and W.Z.) of the University Hospital of Basel, Switzerland, between September 1987 and July 1990. All cases were referred by the orthopedic surgeon (A.G. or

Received 18 October 1991; revised 3 February 1992.

This work was presented in part at the 31 st Interscience Conference on Antimicrobial Agents and Chemotherapy, held in 1991 in Chicago.

A.F.W. is the recipient of an award of the Swiss National Science Foundation (no. 27922).

* Present address: Department of Medicine, Division of Clinical Epidemiology, University of Iowa Hospitals and Clinics, C-41 GH, Iowa City, lowa 52242.

Reprints or correspondence: Dr. Andreas F. Widmer, Department of Internal Medicine, Kantonsspital Basel, Petersgraben 10, CH-4031, Basel, Switzerland.

Clinical Infectious Diseases 1992;14:1251-3

(C) 1992 by The University of Chicago. All rights reserved.

$1058-4838 / 92 / 1406-0010 \$ 02.00$
P.E.O.) who decided not to remove the implant because it was needed for stabilization of a fracture or because it was considered to be difficult to remove. Patients included in the study met the following criteria for ODRI: (1) local pain; (2) local signs of inflammation, and (3) isolation of gram-positive cocci from joint aspirates or intraoperative tissue that were susceptible to rifampin. Included patients were periodically examined at least weekly during the first month of infection and at least monthly thereafter. Patients with internal fixation devices were observed every 3 months once clinical cure was established for $>1$ year (cases $1-5$ and 11 , table 1 ). For patients with prostheses at least bimonthly clinical visits were continued until the time of this writing ( $>24$ months, cases 7-10) or until they died (case 6).

All 11 patients were treated with the implant in situ by the standard antimicrobial regimen initiated by the attending physician. After inclusion into the study, the patients received their current antimicrobial regimen plus rifampin. There was no control group because the orthopedic surgeon referred patients only if the current state-of-the-art therapy was most likely to fail. A treatment course of at least 2 months was planned. The upper limit of duration of treatment was determined by the clinical and laboratory signs of infection.

Patients were considered to be clinically cured if they suffered from no or minimal pain, returned to the level of activity prior to the infection, and did not show signs of infection on physical examination. Laboratory findings indicating cure were defined as an erythrocyte sedimentation rate of $<30 \mathrm{~mm} / \mathrm{h}$ or a C-reactive protein concentration of $<20 \mathrm{mg}$ / $\mathrm{L}$ and a white blood count of $<10,000 / \mathrm{mm}^{3}$. Endpoints of the study were defined as follows. Success was defined as 
Table 1. Summary of data on characteristics and outcome of 11 patients with orthopedic implant-related infections due to staphylococci or streptococci.

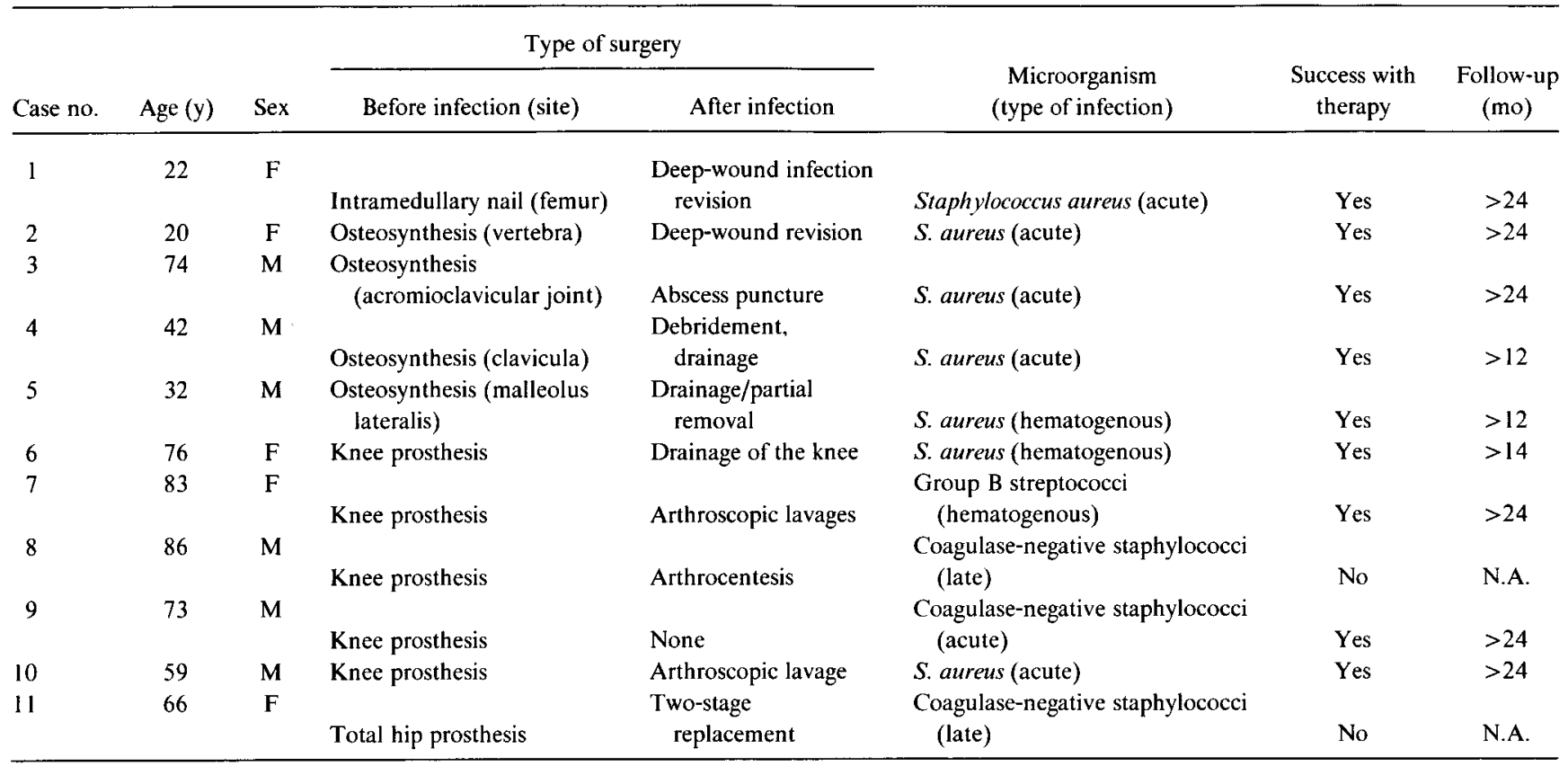

NOTE. N.A. = not applicable.

clinical cure and laboratory parameters indicating no signs of infection $>24$ months after cessation of antimicrobial therapy. Success was also defined as patient survival or no scheduled removal of the internal fixation devices $>6$ months after clinical cure. If the internal fixation devices were removed, negative results of culture of the whole device were required. Failure was defined as the presence of clinical and/or laboratory signs of infection after cessation of antimicrobial therapy. Antimicrobial therapy was guided by the MIC for the infecting strain [6] and the presence or absence of $\beta$-lactamase production. The antimicrobial regimen for all patients except one (case 11) included rifampin within 6 days after the start of antimicrobial therapy. Patient 11 received monotherapy with ciprofloxacin before being referred to the Infectious Disease Service and being included in the study.

The median age was 66 years (table 1). Infections were related to osteosynthesis material (cases 1-5), knee prosthesis (cases 6-10), and hip prosthesis (case 11). Six patients had acute implant-related infection after surgery, three had hematogenous infection (case 5, during sporadic use of intravenous heroin; case 6 , with no obvious source; and case 7 , following teeth extraction), and two had delayed infection with 6- and 9-month duration of symptoms before administration of the present antimicrobial therapy.

Treatment was successful for nine $(82 \%)$ of 11 patients (table 1). Failure of therapy was observed for two patients who had delayed infection and for whom antimicrobial treatment was considered inappropriate. The excellent success rate in our study (82\%) confirms data from in vitro and ani- mal experiments $[3,4,7,8]$. A recent study showed that a cure rate of $90 \%$ can be achieved with combination therapy with ofloxacin and rifampin [9]. Rifampin has excellent efficacy in the treatment of ODRIs (these infections may be cured), a property that correlates with its ability to eradicate adherent and nongrowing staphylococci. Combination therapy with ciprofloxacin and rifampin has recently been used effectively as oral treatment of $S$. aureus right-sided endocarditis [10]. Rifampin has good bioavailability, a relatively long half-life ( 2.5 hours), and excellent activity against staphylococci. However, because of the frequent emergence of organisms that are resistant to rifampin, a second drug is required for treatment of ODRIs.

The design of our study, the small number of cases, and the lack of controls limit the external validity of the results. However, the orthopedic surgeons referred patients to the Infectious Disease Service only when their treatment options were most likely to fail, thus introducing a potential bias toward findings that contradict those of our own. Furthermore, this therapy does not preclude regular treatment, including removal of the device if treatment with rifampin combinations fail. On the basis of these clinical results and the encouraging data from in vitro tests and animal experiments [3-5], a multicenter, double-blind controlled clinical trial comparing regular antimicrobial treatment with and without rifampin was launched in January 1992. However, with the low incidence of such infections and the required follow-up, the results of the study will not be published before 1996. Until such data are available, the results of the clinical study 
presented herein (supported by in vitro data and animal experiments) may contribute to the treatment options for current patients for whom removal of the implant is not feasible.

\section{References}

1. Fitzgerald RH Jr. Infections of hip prosthesis and artificial joints. Infect Dis Clin North Am 1989;3:329-38.

2. Dellamonica $P$, Etesse-Carsenti $H$, Bernard E, Mondain V, Durant $J$, Argenson CI. Pefloxacin in the treatment of bone infections associated with foreign material. J Antimicrob Chemother 1990;26 (suppl B): 199-205.

3. Widmer AF, Frei R, Rajacic Z, Zimmerli W. Correlation between in vivo and in vitro efficacy of antimicrobial agents against foreign body infections. J Infect Dis 1990;162:96-102.

4. Tshefu K, Zimmerli W, Waldvogel FA. Short-term administration of rifampin in the prevention or eradication of infection due to foreign bodies. Rev Infect Dis 1983;5(suppl 3):S474-80.

5. Zimmerli W, Zak O, Vosbeck K. Experimental hematogenous infection of subcutaneously implanted foreign bodies. Scand J Infect Dis 1985; 17:303-10.

6. Lorian V, Burns L. Predictive value of susceptibility tests for the outcome of antibacterial therapy. J Antimicrob Chemother 1990;25: 175-81.

7. Zimmerli W, Waldvogel FA, Vaudaux P, Nydegger UE. Pathogenesis of foreign body infection: description of an animal model. $\mathbf{J}$ Infect Dis 1982;146:487-97.

8. Widmer AF, Wistner A, Frei R, Zimmerli W. Killing of non-growing and adherent Escherichia coli determines drug efficacy in device-related infections. Antimicrob Agents Chemother 1991;35:741-6.

9. Raoult D, Drancourt M, Argenson JN, Aubaniac JM. Rifampin plus ofloxacin for treatment of staphylococcal-infected hip- and kneeprosthesis. [abstract no 1281]. In: Program and abstracts of the 31st Interscience Conference on Antimicrobial Agents and Chemotherapy. Washington, DC: American Society for Microbiology, 1991.

10. Dworkin RJ, Lee BL, Sande MA, Chambers HF. Treatment of rightsided Staphylococcus aureus endocarditis in intravenous drugs users with ciprofloxacin and rifampicin. Lancet 1989;2:1071-3. 